\title{
High speed digital imaging of cavitating vortices
}

\author{
K. R. Laberteaux, S. L. Ceccio, V. J. Mastrocola, J. L. Lowrance
}

\begin{abstract}
Researchers at the Cavitation and Multiphase Flow Laboratory of the University of Michigan worked in conjunction with Princeton Scientific Instruments (PSI) engineers to employ a new digital imaging system in the study of partial attached cavitation. The new high speed solid state system, the Princeton Scientific Ultra Fast Framing Camera (UFFC), was designed for cavitation studies where framing rates of $10^{5}-10^{6}$ frames/s are required to image the detailed mechanisms of cavitating flows. The UFFC, which uses a PSI patented Charge Coupled Device (CCD) array image sensor, was designed to capture 30 frames at a maximum framing rate of 1 million frames/second. In these experiments, a maximum framing rate of 125000 frames per second ( $8 \mu \mathrm{s} /$ frame) was used to examine cavitating vortices in the closure region of a partial attached cavity. The vortical structures in the closure region of the attached cavity were imaged, and the evolution and collapse of these flow structures were examined. Relationships between the cavitating vortices size, strength, and collapse time were observed.
\end{abstract}

\section{1}

\section{Introduction}

High speed photography has often been used to study the micro fluid mechanics of cavitating flows. Researchers have visualized complex cavitating flows to increase understanding of the inception and evolution of cavitation. Flash photography is often used to capture a single image of the cavitation. However, single images cannot record the complex evolution

\section{Received: 15 November 1996/Accepted: 1 December 1997}

\section{K. R. Laberteaux, S. L. Ceccio}

Department of Mechanical Engineering and Applied Mechanics University of Michigan, Ann Arbor, Mi 48109-2121, USA

V. J. Mastrocola, J. L. Lowrance

Princeton Scientific Instruments, Inc.

Monmouth Junction, NJ 08852, USA

Correspondence to: S. L. Ceccio

The authors would like to thank Ann Tassin-Leger, Darin George, and Noah Shirk for their assistance during the experiments. This research was supported by Office of Naval Research contract N00014-96-1-0076. Development of the Princeton Scientific Ultra Fast Framing Camera was supported by Office of Naval Research contract N0014-91-0-0078. Dr. Edwin P. Rood is technical coordinator for both contracts. of cavitation since most cavitating flows are unsteady with randomly occurring events. For example, the closure region of a partial, stable, attached cavity is characterized by a large number of cavitating vortices and bubbles. These flow structures evolve and collapse randomly.

Avellan et al. (1988) discussed the formation of cavitating vortices in the cavity closure region. It was suggested that the "hair-pin" like vortices resulted from Kelvin-Helmholtz type instabilities of nominally spanwise vorticity in the flow above the cavity surface. Researchers have also examined cavitating wakes behind bluff objects, which is qualitatively similar to the flow behind a partial closed cavity. Young and Holl (1966), Franc and Michel (1983) and Belahadji et al. (1994) examined the cavitating turbulent wake behind wedges. Katz and O'Hern (1986) and O'Hern (1990) studied the cavitating shear flow behind a sharp-edged plate, and Katz (1984) examined incipient cavitation in the separated flow over bluff axisymmetric bodies. These researchers have found that streamwise cavitating vortices are often the first to be observed with a reduction in cavitation number. The inception of spanwise vortices occur at lower pressures. While the specific inception index of any flow will be strongly influenced by the freestream nuclei content, it appears the pressure in the cores of secondary streamwise vortices in cavitating wakes is generally less than the core pressure of the primary spanwise vortices in cavitating shear layers. A general observation emerged that cavitation inception occurs within organized vortical structures in the cavitating shear flow. Often the structure of the non-cavitating shear layer is visualized by the incipient cavitation. O'Hern (1990), for example, demonstrated a correspondence between the spanwise (primary) and streamwise (secondary) vortices which occurred in both free shear layers and in an incipient cavitating wake.

The presence of significant amounts of cavitation in the wake of an attached cavity will significantly modify the organization of vorticity. The study of cavitating vortical flows is then much more complex. Single images from flash photography cannot record the complex evolution of cavitating vortical structures since they are randomly occurring events. A time series of images is necessary to determine if the vortices are simply advecting with the mean flow without volume changes, or if they are evolving and collapsing. High speed motion pictures offer a solution.

Film cameras employing rotating prisms and film on rotating drums can achieve framing rates up to $10^{6}$ frames/s. Such high framing rates are necessary to capture cavitation transients. Avellan and Farhat (1989) examined vortices 
"on demand" through the use of a vortex generator. Images of the collapsing vortex were captured on film using a Cranz-Schardin camera. However, high speed film cameras have severe limitations. Synchronization of the camera operation with naturally occurring cavitation events can be problematic. Also, a large amount of illumination is required, especially at high image magnification. The greatest drawback to film cameras is the delay between the image capture and the development of the final image, which can complicate camera setup.

High speed videography offers several advantages over film. These cameras record an electronically captured image. The illumination requirements of these cameras are often much lower than those of film cameras. In addition, video recording permits the capture and playback of images during a single experimental session. However, the resolution of these cameras is often two orders of magnitude less than that of photographic film. The spatial resolution of commercial high speed digital video systems is on the order of $256 \times 256$ pixels. Moreover, the maximum framing rate of such cameras is $\sim 10^{4}$ frames/s, which is not sufficient to capture detailed images of cavitation.

In order to capture images of transient cavitating flow structures, a camera with a much higher framing rate is needed. Such a ultra-high speed digital camera has been developed by Princeton Scientific Instruments (PSI). The high speed solid state imaging system, which uses a PSI patented Charge Coupled Device (CCD) array image sensor, was designed to capture 30 frames at a maximum framing rate of 1 million frames/s. The PSI Ultra Fast Framing Camera (UFFC) was designed for cavitation studies where framing rates of $10^{5}-10^{6}$ frames/s are required to image the detailed mechanisms of cavitating flows.

Researchers at the Cavitation and Multiphase Flow Laboratory of the University of Michigan worked in conjunction with PSI engineers to employ this new digital imaging system in the study of partial attached cavitation. The vortical structures in the closure region of the attached cavity were imaged, and the evolution and collapse of these flow structures were examined. Thus, the capabilities of the UFFC were explored in the laboratory environment.

\section{2}

\section{Experimental setup}

The experiments described here were conducted in the Cavitation and Multiphase Flow Laboratory at the University of Michigan, Department of Mechanical Engineering and Applied Mechanics. The experimental setup is described in the following sections.

\section{1}

\section{Model large cavitation channel and cavitation model}

The Model Large Cavitation Channel (MLCC) is a small recirculating water tunnel in which steady freestream flows are used to study cavitation (George and Ceccio 1995). The MLCC volume is approximately $2.8 \mathrm{~m}^{3}$. The tunnel is connected via control valves to a vacuum reservoir, and the pressure in the test section can be reduced from near atmospheric pressure to vacuum. The rectangular test section has interior dimensions of $21.6 \times 21.6 \times 118.1 \mathrm{~cm}$, with a cross sectional area of $446 \mathrm{~cm}^{2}$. Before experiments, the air content of the water can be reduced from saturation to approximately $3 \mathrm{ppm}$ oxygen content by circulation between the MLCC and a $2.3 \mathrm{~m}^{3}$ deaeration tank. Flow in the MLCC is produced by an impeller in the bottom leg, driven by a 150-horsepower AC motor with an eddy current speed controller.

A two-dimensional bi-convex hydrofoil with a rounded leading edge is used in the experiments. Figure 1 presents a photograph of the cavitating hydrofoil. The area imaged by the UFFC is marked. The maximum thickness of the hydrofoil is $1.3 \mathrm{~cm}$, the chord is $14.8 \mathrm{~cm}$, and the leading edge radius of curvature is $0.16 \mathrm{~cm}$. The hydrofoil cross section is shown in Fig. 2. The foil was constructed from free-machining brass and highly polished. The foil is mounted between two opposing Lucite windows to permit variation in the angle of attack. The angle of attack is approximately $+2.5^{\circ}$ to achieve a developed, stable cavity.

\section{2}

\section{PSI Ultra Fast Framing Camera}

The Princeton Scientific Instruments (PSI) Ultra Fast Framing Camera is a high speed solid state imaging system that uses a PSI patented CCD array image sensor. This image sensor provides an on-chip storage of 30 frames. The charge from a photodetector is shifted out serially for each of five frames, followed by a vertical shift that moves the entire row. The previously stored charge from older images is dumped and replaced by the charge from new images. In these experiments, the CCD array is exposed at a maximum rate of 125000 frames/s ( $8 \mu \mathrm{s} /$ frame). The CCD image rastor is $180 \times 180$ pixels. An external trigger (see Sect. 2.4) is used to stop the image acquisition. After image acquisition, the series of images are retrieved and stored in approximately $10 \mathrm{~s}$.

The Ultra Fast Framing Camera main components are the Camera Head, the Control Chassis, and the Data Acquisition System. See Fig. 3 for a schematic of the PSI imaging system. The Camera Head is a vacuum enclosure that houses the CCD array and a thermo-electric cooler. The CCD array operating temperature is approximately $-30{ }^{\circ} \mathrm{C}$. The thermo-electric cooler provides cooling of the CCD assembly, in combination with circulating water that carries heat away from the hot side of the Camera Head. A mechanical shutter is incorporated in front of the CCD, but outside of the vacuum enclosure. The shutter is opened prior to exposure and closed during the readout on command from the Control Chassis. A lens is positioned in front of the shutter.

The Control Chassis is a $101 / 2^{\prime \prime} \times 19^{\prime \prime}$ card cage containing power supplies and electronics for control and operation of the camera head. The frame rate, trigger mode, and other options can be controlled through the Data Acquisition Computer. Clocking signals and bias voltages are sent to the Camera Head and video signals are returned to the Control Chassis. A video processing board amplifies the video signal and performs double correlated sampling to reduce noise and maximize dynamic range. The video signal is digitized and sent to the Data Acquisition and Control Computer via opto-isolated serial links.

An Intel 486 based computer receives the serial digital data from the Control Chassis and stores it in memory. Each line of 

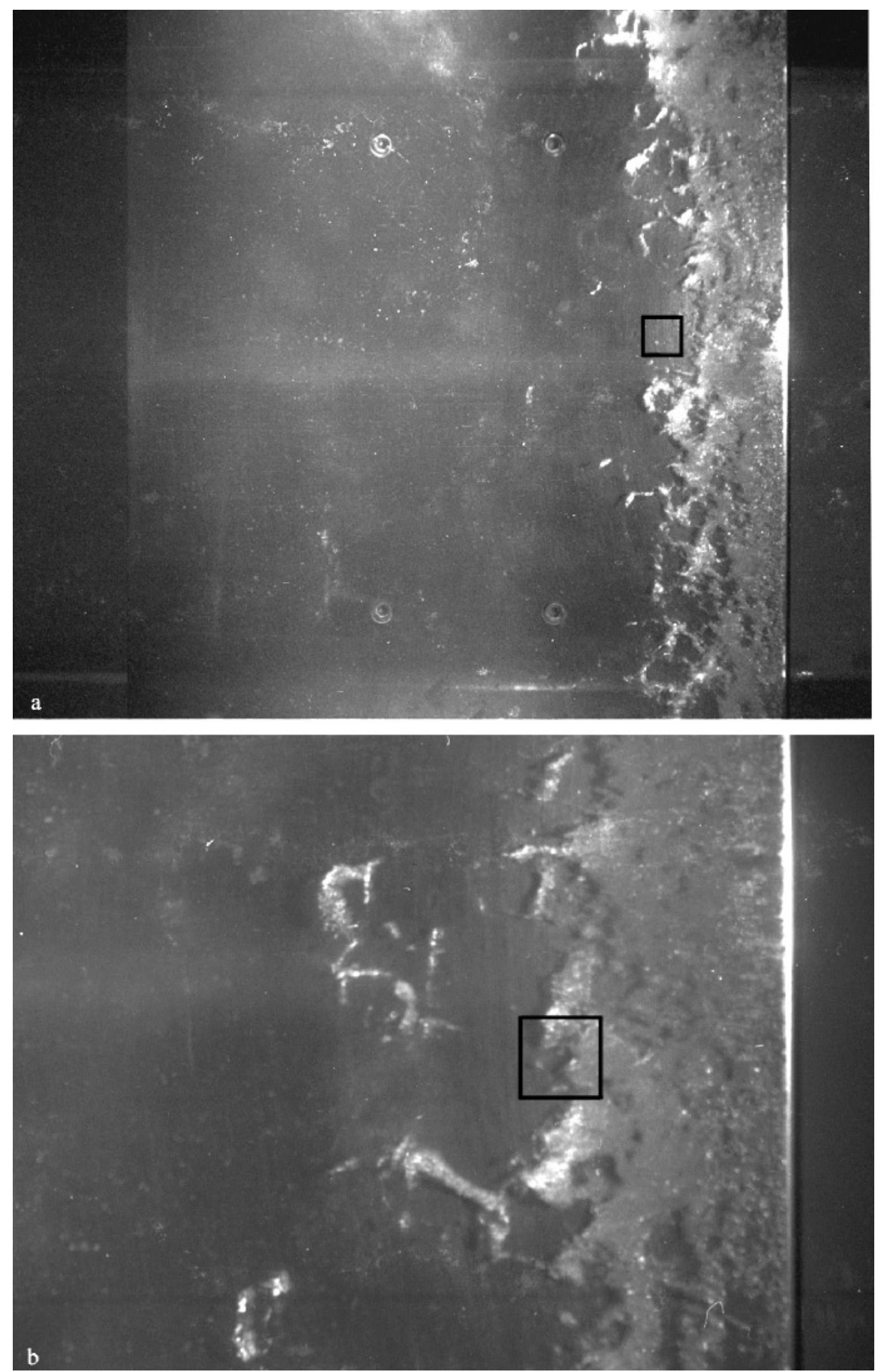

Fig. 1. Photograph of cavitating hydrofoil in the MLCC. The $9 \mathrm{~mm}$ square interrogation area imaged by the UFFC is marked

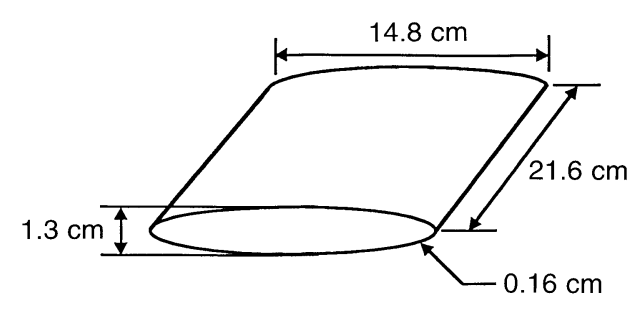

Fig. 2. Schematic diagram of the bi-convex hydrofoil used during experiments the data contains pixels from five different frames. As this data is read into memory, it is sorted such that pixels from a given frame are stored together and in sequential order. The data is formatted as digitized images that are stored as 30 separate files in FITS format. A video monitor and interface board provide a video display of the images in memory. Images can be stepped through one at a time, or played in a continuous loop. Images previously stored on the hard disk can be reloaded into memory and displayed on the video monitor. 


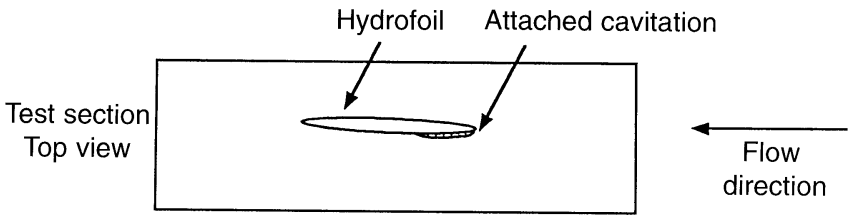

Camera

head
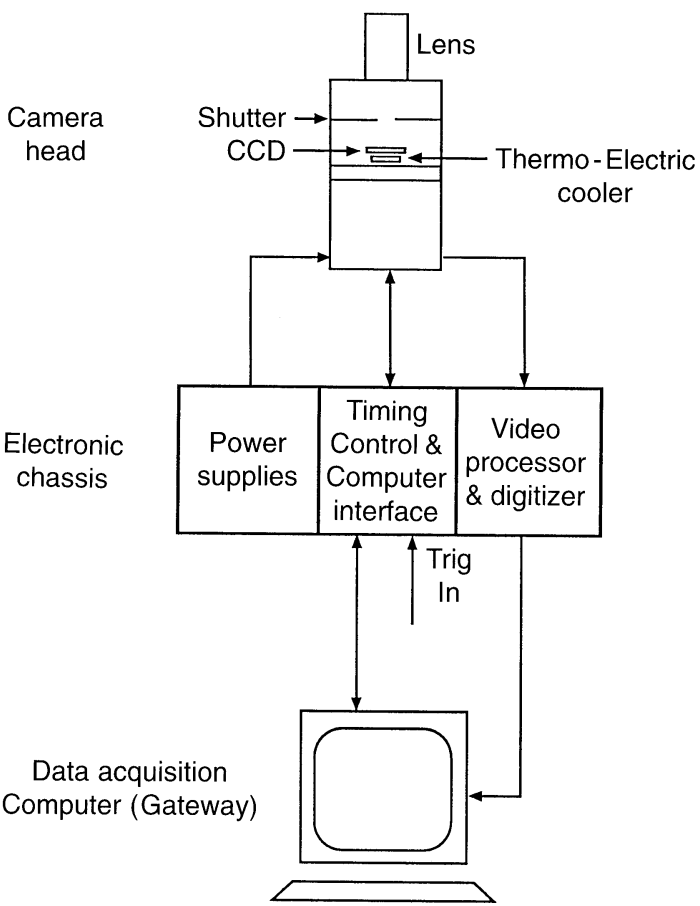

Fig. 3. Schematic of the imaging setup. The PSI Ultra Fast Framing Camera system is directed at the test section of the MLCC

\section{3}

\section{Illumination system}

A continuous light beam produced with a Lexel Model 95, $4 \mathrm{~W}$ Argon-Ion laser (wavelength $514 \mathrm{~nm}$ ) is used as a monochromatic light source. A plano-concave lens (focal length $=$ $-150 \mathrm{~mm}$ ) is used to expand the $1.3 \mathrm{~mm}$ diameter laser beam (see Fig. 4). In addition to the laser light, a focused $750 \mathrm{~W}$ incandescent light source is used to provide additional illumination with full spectrum frequency. The flow was illuminated with different combinations of light to obtain maximum illumination with minimum IR leakage to the CCD.

The ultra fast framing CCD image sensor was found to have a problem of parasitic leakage of photons and/or photoelectrons from the photodetector area into the memory array portion of the pixel. This signal leakage into exposed images amounts to approximately $1 \%$ per exposure in the outer columns of the $5 \times 6$ memory array for red light, and about $0.4 \%$ in the green light. While not understood in detail, this suggests that the red photons, which have a longer absorption path length through the silicon, are more easily transported/reflected into the adjacent memory regions. This problem has been addressed in the next lot of CCDs. However, this problem had to be contended with in the current cavitation images.

\section{4}

\section{Event triggering system}

A triggering system is implemented to detect cavitating vortical structures as they passed through the camera field of view. The beam of a $1 \mathrm{~W} \mathrm{He}-\mathrm{Ne}$ laser is reflected off the foil and the reflected beam was directed into a photo diode (see Fig. 5). When a cavitating bubble passed across the laser beam, light is prevented from reaching the photo diode, producing a signal. The output of the photo-diode is filtered to eliminate spurious signals, a trigger delay is added, and the signal is conditioned to trigger the camera.

For triggered operation, the camera runs with the shutter open in a continuous erase/expose mode. In this mode, all frames in the storage area are continually overwritten with new images. When a trigger is received, the overwriting is stopped. Thus, the most recent 30 frames prior to the

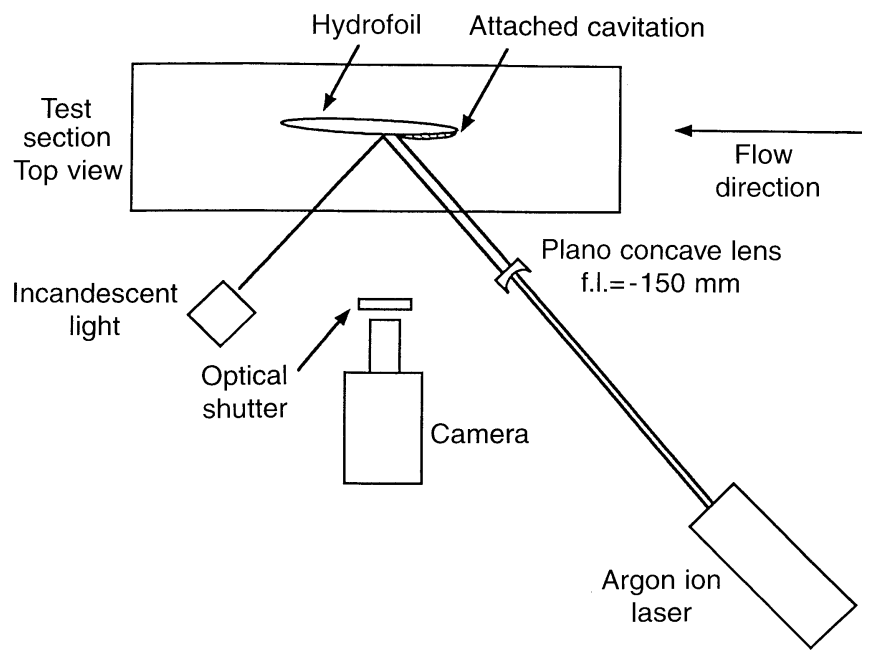

Fig. 4. Schematic diagram of the illumination system

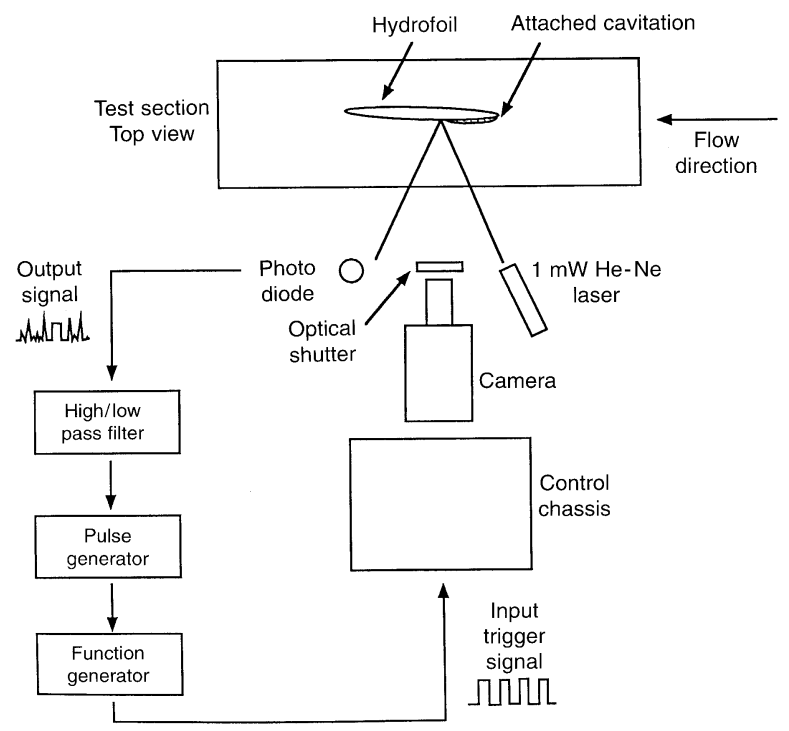

Fig. 5. Schematic diagram of the cavitation event triggering system 
trigger are stored. The shutter then is closed, and the data is recorded.

It was important to turn off the illumination once the images had been acquired to minimize the signal leakage effect (see Sect. 2.3). An Oriel 50075 Optical Shutter was used in conjunction with the signal processing to eliminate light "bleed" into the camera. The electro-optical shutter is placed directly in front of the camera lens and is used to block the aperture into the Camera Head microseconds after the camera finishes image acquisition. The addition of this shutter led to only a partial reduction of light leakage. Excessive light leakage made image acquisition difficult at the maximum framing rate. As a result, the maximum framing rate during these experiments is $8 \mu \mathrm{s} /$ frame.

\section{3}

\section{Results and discussion}

The experimental system described above was used to examine the cavitating vortices in the closure region of a stable, partial, attached cavity. The growth, collapse, and rebound of these cavitation bubbles were recorded. The cavity closure region is characterized by a large number of cavitating vortices and bubbles. Magnified images of these flow features were examined. During the experiments, the magnitude of the freestream velocity, $U_{\infty}$, was $10 \mathrm{~m} / \mathrm{s}$, and the freestream cavitation number $\sigma_{\infty}=\left(P_{\infty}-P_{V}\right) / \frac{1}{2} \rho U_{\infty}^{2}=1.64$, where $P_{\infty}$ is the freestream static pressure, $P_{V}$ is the liquid vapor pressure, and $\rho$ is the liquid density. The dissolved oxygen content was approximately $5 \mathrm{ppm}$ at atmospheric pressure.

Figure 6 through 9 present a series of 30 images taken by the UFFC. The images in Figs. 6 and 7 were acquired at a rate of $32 \mu \mathrm{s} /$ frame, and those of Figs. 8 and 9 were acquired at $8 \mu \mathrm{s} /$ frame. The first frame of the sequence is at the top left corner of the figure. The time sequence progresses from left to right, top row to bottom row, where the last frame is on the bottom right corner. In each frame, the flow direction is from right to left. The field of view on each frame is $9 \mathrm{~mm}$ square. In Figs. 6 and 7, cavitating vortices are observed collapsing, rebounding, and collapsing again. In Figs. 8 and 9, a cavitating vortical structure is collapsing. A large number of such image sequences were acquired for randomly passing cavitation structures. Quasi-spherical cavitation bubbles, vortical bubble "tubes", and collections of small cavitation bubbles were observed. The cavitating vortices examined in the flow behind the partial cavity were observed to be straight or crescent shaped cavitating segments. The vortices were not easily divided into streamwise and spanwise coherent structures. By examining a time series of images, it was possible to determine if the vortices were simply advecting with the mean flow without volume changes, or if they were collapsing.

The Rankine model used by O'Hern (1990) to examine streamwise vortices can be used here to estimate the strength of the cavitating vortices in the cavity closure. O'Hern (1990) observed that the streamwise vortices incepted before the spanwise vortices. However, he also showed that strength of the cavitating streamwise vortices, $\Gamma$, was generally less than $10 \%$ of the strength of cavitating spanwise vortices. A Rankine vortex was employed in O'Hern's studies to model the streamwise vortices, with $\Gamma=\pi \omega R_{C}^{2}$, where $\omega$ is the vorticity, $R_{C}$ is the vortex core radius. The pressure within the core of the
Rankine vortex, $P_{C}$ is given by

$P_{C}=P_{\infty}-\rho\left(\frac{\Gamma}{2 \pi R_{C}}\right)^{2}$

It was assumed that the core of the cavitating Rankine vortex was filled with vapor, and thus the local cavitation number, $\sigma_{L}$, and local flow speed, $U_{L}$, can be related to the vortex strength

$\Gamma=2 \pi R_{C} U_{L} \sqrt{\frac{1}{2} \sigma_{L}}$

The strength of the spanwise vortices was found by examining 493 the streamwise spacing of the vortices and the velocity difference across the shear layer.

While the actual cavitating vortical flow is much more complex than this analysis suggests, basic relationships between the local flow conditions, the size of the cavitating vortex, and the vortex strength can be found using these ideas. The radius of non-collapsing cavitating vortices were measured along with their orientation to the mean flow direction. By using the local coefficient of pressure, $C_{P L}=P_{L}-P_{\infty} / \frac{1}{2} \rho U_{\infty}^{2}$, the vortex strength, $\Gamma$, is estimated using the following relationship:

$\Gamma=2 \pi R_{C} U_{L} \sqrt{\frac{1}{2} \sigma_{L}}=2 \pi R_{C} U_{\infty} \sqrt{\frac{1}{2}\left(C_{P L}+\sigma_{\infty}\right)}$

Le et al. (1993) presented the pressure coefficient distribution, $C_{P}$, around partial cavities occurring on a plano-convex foil. They showed that the pressure coefficient in the cavity closure region ranged from $-\sigma_{\infty}$ to approximately zero. We can therefore estimate that $C_{P L} \approx-\frac{1}{2} \sigma_{\infty}$. Thus,

$\Gamma=\pi R_{C} U_{\infty} \sqrt{\sigma_{\infty}}$

Figure 10 presents a plot of $\Gamma$ as a function of vortex orientation. $\theta$ is the magnitude of the angle the vortex made with the mean flow direction. If $\theta=0^{\circ}$, the cavitating vortex was oriented in the streamwise direction, and if $\theta=90^{\circ}$, the cavitating vortex was oriented in the spanwise direction. Spanwise cavitating vortices were consistently observed to have a larger radius compared with streamwise cavitating vortices, and thus the estimated strengths of the spanwise vortices are larger. The radius (strength) of the vortices appears to be proportional with the orientation angle. It is important to note that a time series of images is necessary to acquire this data, since we have only considered cavitating vortices which are not growing or collapsing.

Collapsing cavitating vortices were also examined. Figure 11 presents a plot of the projected area of a collapsing vortex as a function of time. These data were derived from images shown in Fig. 6. Note that the cavity grows, reaches a maximum volume, collapses, and rebounds. Almost every collapsing cavity was observed to rebound, and this indicates that non-condensable gas was present within the cavity (along with water vapor). Non-condensable gas diffuses into the attached partial cavity and can be entrained into the wake vortices as they form near the cavity trailing edge (Yu and Ceccio 1996). Also, a nucleus may be captured by a vortex and cavitate. Non-condensable gas may then diffuse into the cavitating vortex core. 


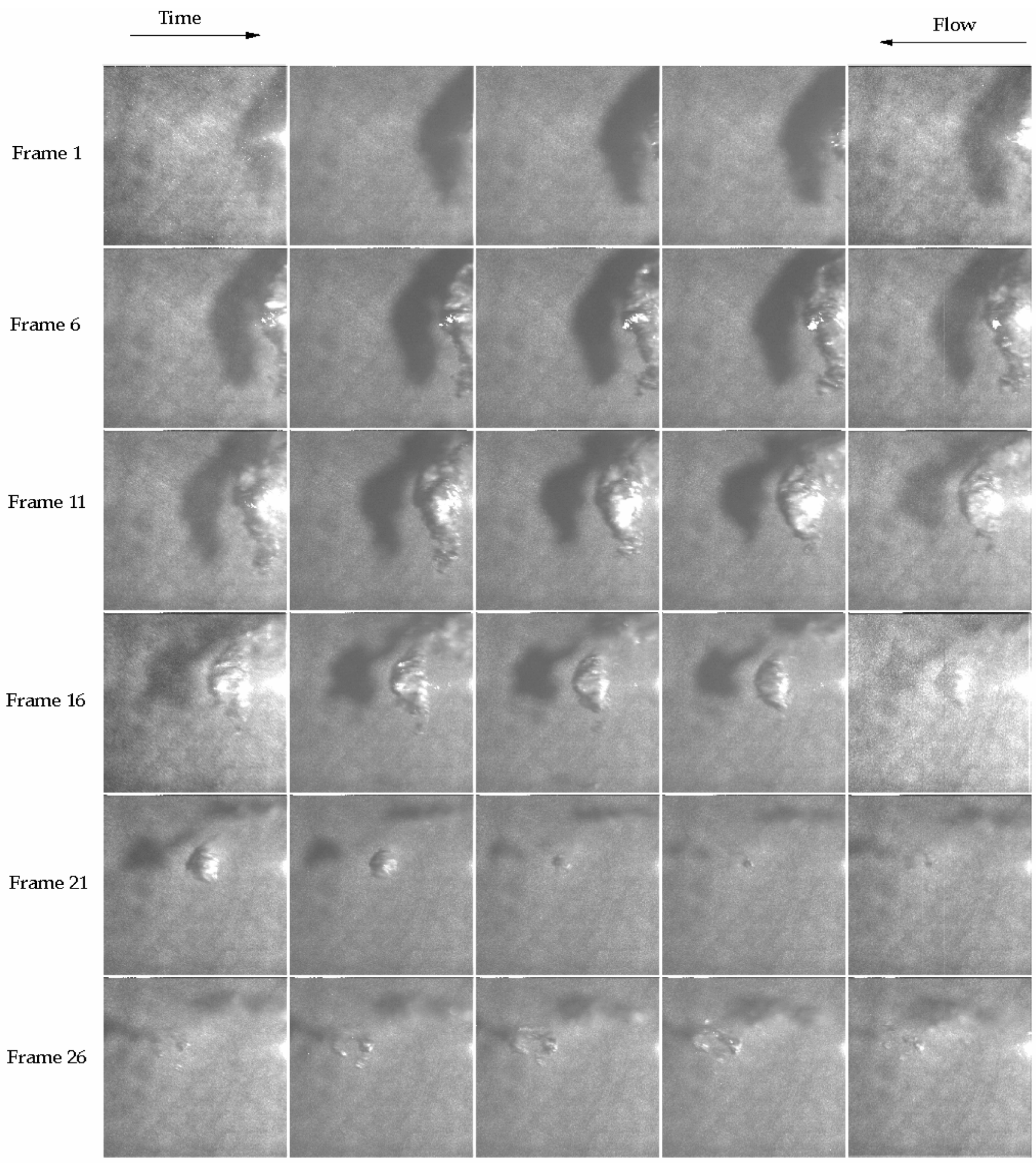

Fig. 6. FFC 30 frame series acquired at $32 \mu \mathrm{s} /$ frame. A cavitating vortical structure collapsing and rebounding. Field of view on each frame is $9 \mathrm{~mm}$ square

The collapse time of the cavitating vortices, $t_{C}$, can be scaled with the Rayleigh collapse time of a spherical bubble

$$
t_{C} \sim \kappa R_{C} \sqrt{\frac{\rho}{P_{L}-P_{V}}}=\frac{\kappa R_{C}}{U_{\infty}} \sqrt{\frac{1}{\frac{1}{2}\left(C_{P L}+\sigma_{\infty}\right)}}
$$

Using the vortex strength, $\Gamma$, equation,

$t_{C} \sim \frac{\kappa R_{C}^{2}}{\Gamma}$

where $\kappa$ is a constant on the order of one. For a spherical bubble, $\kappa=0.915$. Figure 12 plots the measured $t_{C}$ versus 


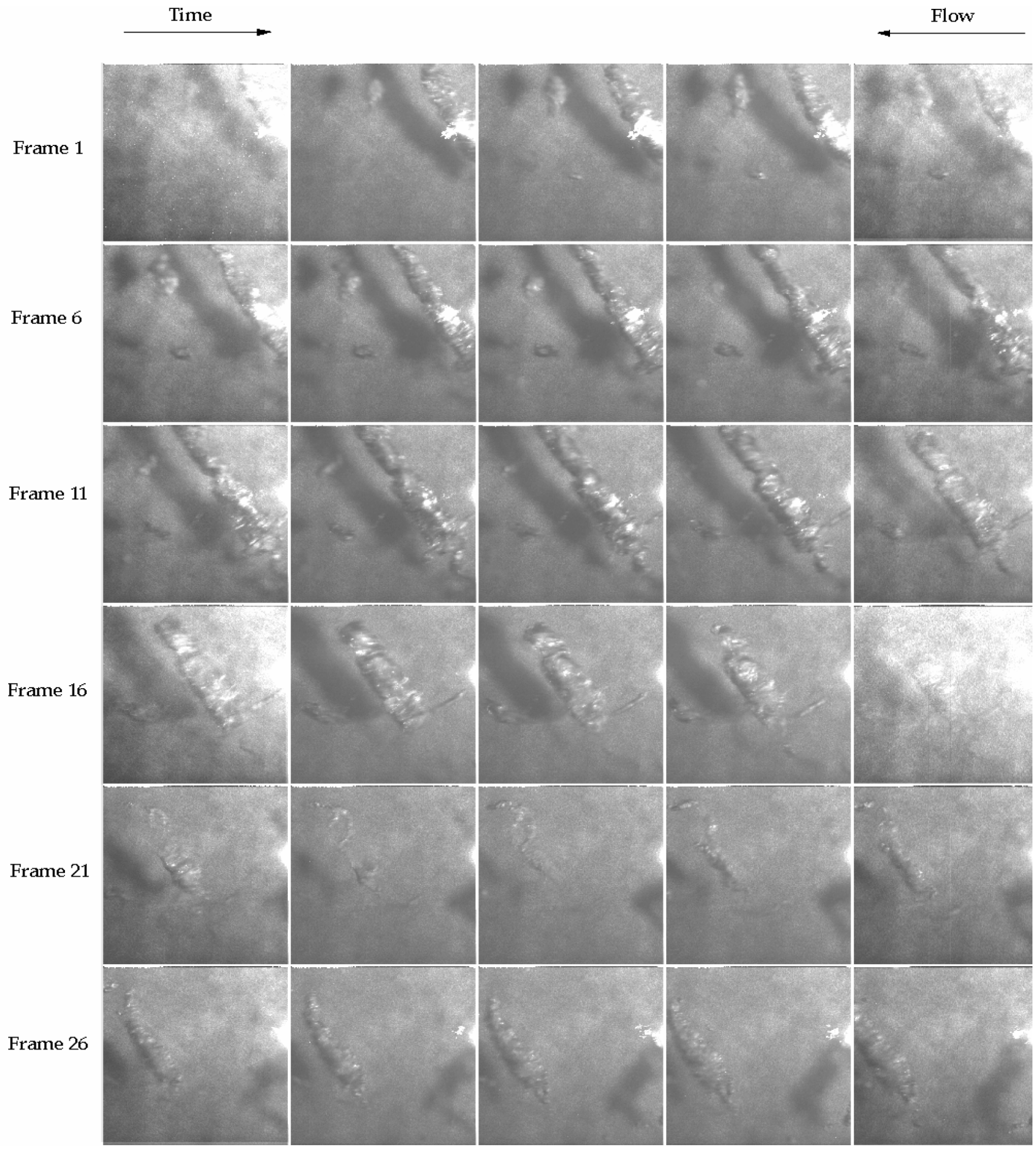

Fig. 7. FFC 30 frame series acquired at $32 \mu$ s/frame. A cavitating vortical structure collapsing and rebounding. Field of view on each frame is $9 \mathrm{~mm}$ square

$R_{C}$ and $R_{C}^{2} / \Gamma$ with the recognition that the estimated vortex strength is proportional to the cavitating vortex radius. The data suggest that the collapse time is proportional to the core size of the cavitating vortex. Chahine and Genoux (1983) analyzed the collapse of a cavitating vortex ring when the ratio of the core diameter to the ring diameter is small $(\varepsilon \ll 1)$. The collapse time of such a vortex ring was also proportional to the Rayleigh collapse time, with $2.0<\kappa<2.3$ for $0.20>\varepsilon>0.05$. Plotted on Fig. 12 are two lines corresponding to the predicted collapse time for $\kappa=0.915$ (spherical bubble collapse) and $\kappa=2.3$ (collapse of a slender vortex ring). Here, the freestream cavitation number and flow velocity were employed, and 


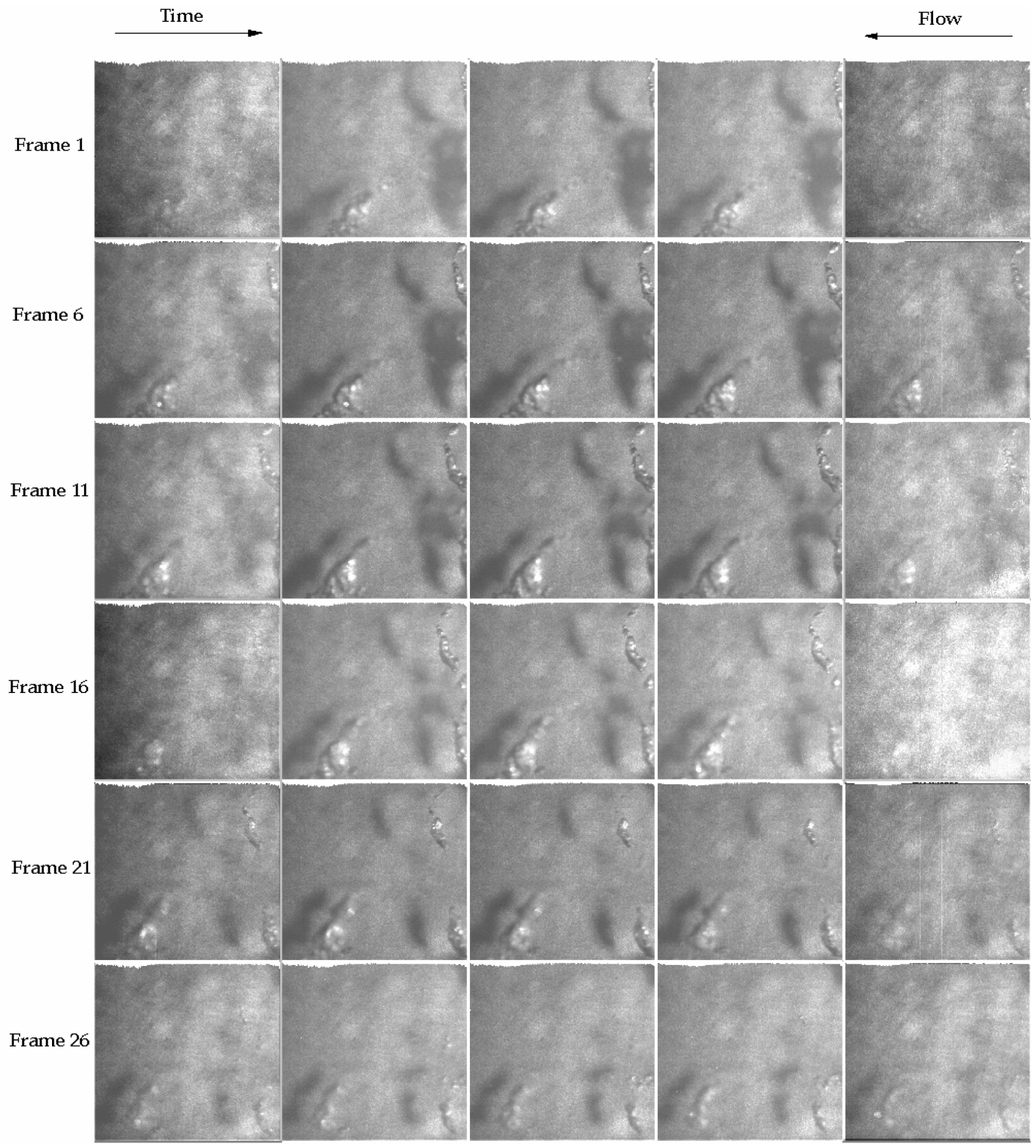

Fig. 8. FFC 30 frame series acquired at $8 \mu \mathrm{s} /$ frame. A cavitating vortical structure collapsing. Field of view on each frame is $9 \mathrm{~mm}$ square

$C_{P L} \approx-\frac{1}{2} \sigma_{\infty}$. The collapse time of the cavitating vortices is bounded by the two lines, which indicates that the collapse of the cavitating vortices is somewhat slower than the collapse of bubbles of radius $R_{C}$, as suggested by Chahine and Genoux (1983). The cavitating vortices collapse faster than a complete vortex ring.

\section{4}

\section{Conclusions}

Cavitating vortices in the closure region of a closed partial attached cavity were examined with a new digital imaging system, the Princeton Scientific Ultra Fast Framing Camera (UFFC). The UFFC, which uses a PSI patented Charge Coupled 


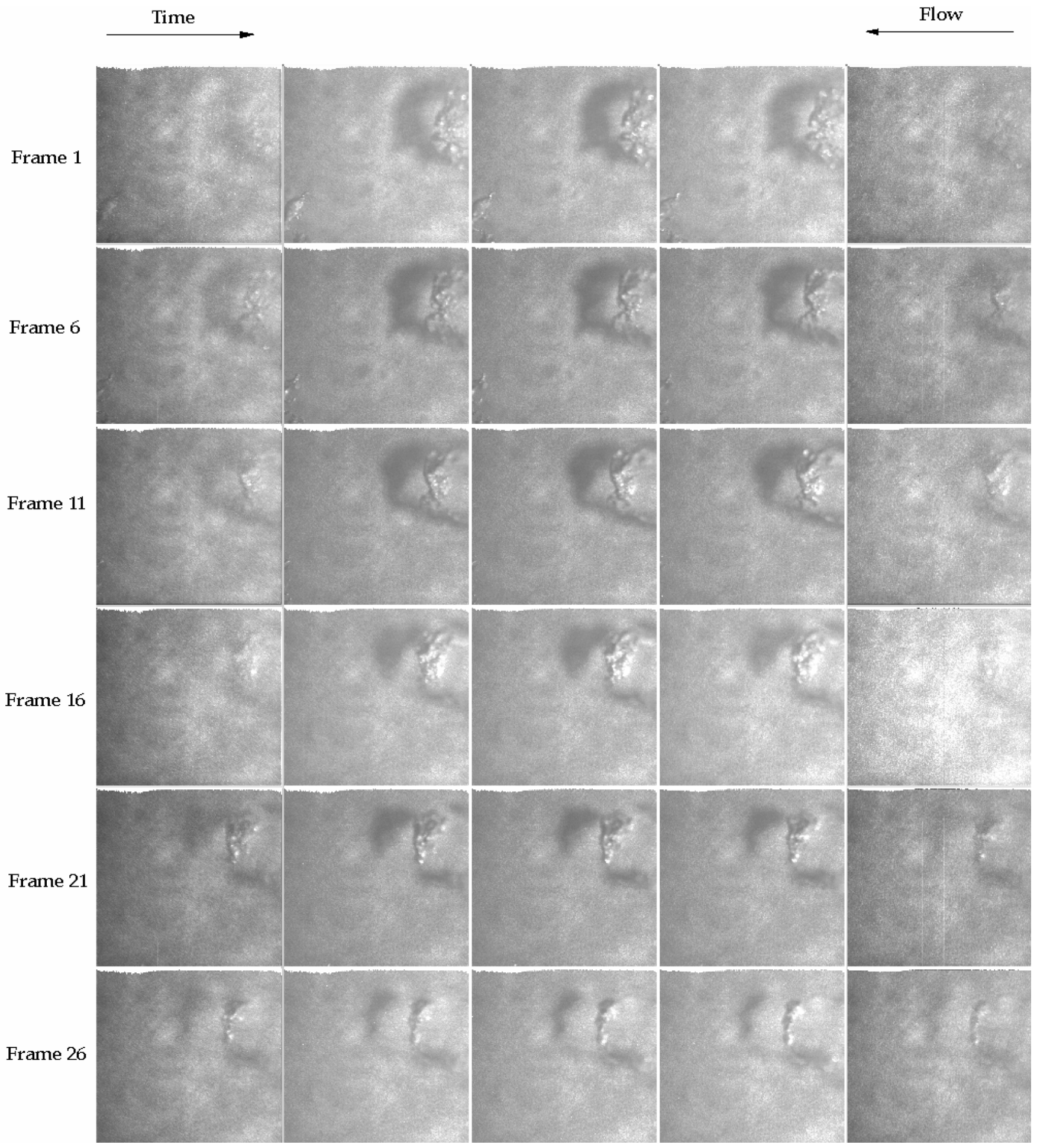

Fig. 9. FFC 30 frame series acquired at $8 \mu$ s/frame. A cavitating vortical structure collapsing. Field of view on each frame is $9 \mathrm{~mm}$ square

Device (CCD) array image sensor, was designed to capture 30 frames at a maximum framing rate of 1 million frames/second. The PSI Ultra Fast Framing Camera (UFFC) was designed for cavitation studies where framing rates of $10^{5}-10^{6}$ frames/s are required to image the detailed mechanisms of cavitating flows. In these experiments, the UFFC framed at a maximum rate of 125000 frames per second $(8 \mu \mathrm{s} /$ frame). The framing rate was limited by the presence of light leakage onto the CCD array. The CCD was originally designed to image 30 frames of $360 \times 360$ pixels, but manufacturing difficulties resulted in a final resolution of $180 \times 180$ pixels.

Magnified images of cavitating vortices were captured by triggering the UFCC. By examining a time series of images, it was possible to determine if the vortices were growing, 


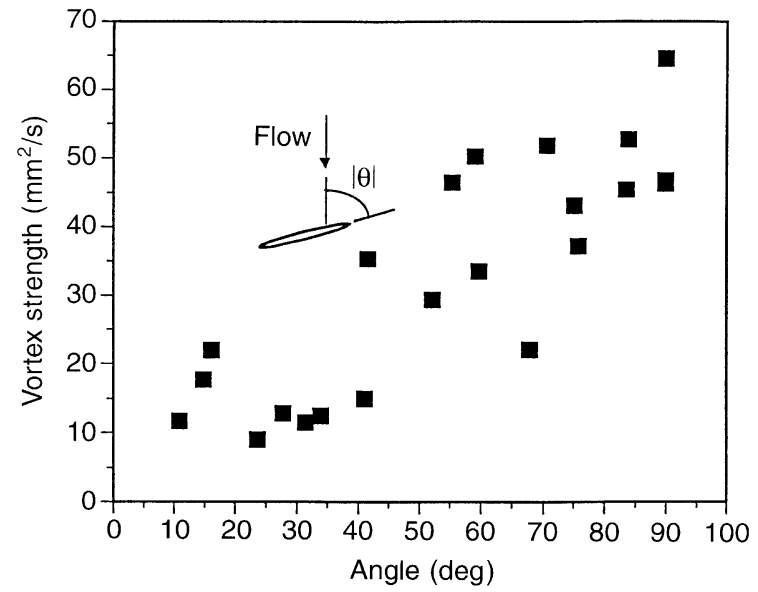

Fig. 10. Vortex strength as a function of vortex orientation. $\theta$ is the magnitude of the angle the vortex makes with the mean flow direction

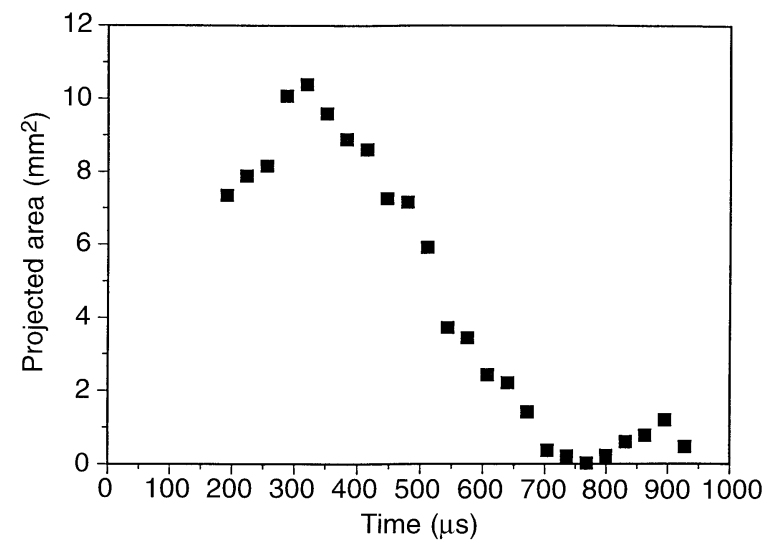

Fig. 11. Projected area of a collapsing vortex as a function of time

advecting with the mean flow, or collapsing. The images were analyzed to estimate the cavitating vortex strength using a simplified Rankine vortex model. The strength of the vortices appeared to be proportional with the orientation angle to the mean flow direction. The collapse and rebound of vortices were examined, and the time a vortex takes to collapse was related to the vortex size.

Princeton Scientific Instruments is developing a version of the Ultra Fast Framing CCD with the same $180 \times 180$ pixel format, but with approximately eight times the sensitivity. The memory capacity is 32 frames and the maximum frame rate will be approximately 2 million frames/s. There are also plans to make an array that will capture images at 10 million frames/s.

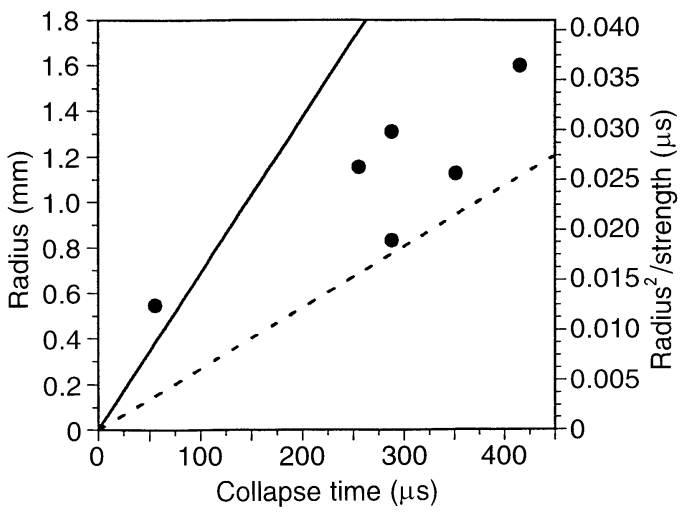

Fig. 12. Cavitating vortex radius as a function of the collapse time. The radius of vortices is measured along their orientation to the mean flow direction. The two lines represent the collapse time of spherical bubbles (solid line) and slender vortex rings with the given core radius (dashed line)

\section{References}

Avellan F; Dupont P; Ryhming I (1988) Generation mechanism and dynamics of cavitation vortices downstream of a fixed leading edge cavity. 17th O.N.R. Symp. on Naval Hydrodynamics, pp. 317-329

Avellan F; Farhat M (1989) Shock pressure generated by cavitation vortex collapse. Proc. A.S.M.E. Int. Symp. On Cavitation Noise and Erosion in Fluid Systems, San Francisco, pp 119-125

Belahadji B; Michel JM; Franc JP (1994) Cavitating vortices in the turbulent wake of a wedge. 2nd Int. Symp. on Cavitation, pp 51-56

Chahine GL; Genoux Ph F (1983) Collapse of a cavitating vortex ring. J Fluids Eng 105: 400-405

Franc JP; Michel JM (1983) Two- and three-dimensional rotational structures in the cavitating turbulent wake of a wedge. Presented at the Fourth Symp. on Turbulent Shear Flows, Karlsruhe, Germany

George DL; Ceccio SL (1995) Cavitation and multiphase flow laboratory at the university of Michigan. Proc. of the 24th American Towing Tank Conference, College Station, Texas, pp 3-9

Katz J (1984) Cavitation phenomena within regions of flow separation. J Fluids Eng 140: 397-436

Katz J; O’Hern TJ (1986) Cavitation in large scale shear flows. J Fluids Eng 108: 373-376

Kosonocky WF; Yang G; Ye C; Lowrance JL; Mastrocola VJ; Shalcross FV; Patel V (1996) $360 \times 360$ element, very high frame rate burst image sensor. 1996 Int. Solid State Circuits Conference, San Francisco, California

Le Q; Franc JP; Michel JM (1993) Partial cavities: global behavior and mean pressure distribution. J Fluids Eng 115: 243-248

Lempert WR; Wu P-F; Zhang B; Miles RB; Lowrance JL; Mastrocola VJ; Kosonocky WF (1996) Pulse-burst laser system for high-speed flow analysis. AIAA 96-0179, 34th Aerospace Sciences Meeting

O'Hern TJ (1990) An experimental investigation of turbulent shear flow cavitation. J Fluids Eng 215: 365-391

Young JO; Holl JW (1996) Effects of cavitation on periodic wakes behind symmetric wedges. Trans. ASME D: J Basic Eng 88D: 163-176

Yu PW; Ceccio SL (1996) Diffusion induced bubble populations downstream of a partial cavity. Proc. ASME Fluids Engineering Division, San Diego, California, pp 345-355 